\title{
Glucose management for exercise using continuous glucose monitoring: should sex and prandial state be additional considerations?
}

\author{
Jane E. Yardley ${ }^{1,2,3,4}$ (D) Ronald J. Sigal ${ }^{5,6,7,8}$ (D) \\ Received: 30 October 2020 / Accepted: 6 November 2020 / Published online: 7 January 2021 \\ (C) The Author(s), under exclusive licence to Springer-Verlag GmbH, DE part of Springer Nature 2021
}

Keywords Blood glucose $\cdot$ Continuous glucose monitoring $\cdot$ Exercise $\cdot$ Hypoglycaemia $\cdot$ Type 1 diabetes

\author{
Abbreviations \\ CGM Continuous glucose monitoring \\ HIIE High-intensity interval exercise
}

To the Editor: In a recent position statement on glucose management for exercise using continuous glucose monitoring (CGM) in type 1 diabetes, Moser et al. reviewed exerciserelated literature and suggested insulin dosage adjustments and carbohydrate intake [1]. We commend the authors for their work, especially considering the lack of strong evidence in the area. Indeed, many suggestions are labelled '(D)', meaning that they originate from expert committee reports and/or expert opinions/clinical experience of respected

Jane E. Yardley

jane.yardley@ualberta.ca

Augustana Faculty, University of Alberta, Camrose, AB, Canada

2 Physical Activity and Diabetes Laboratory, Alberta Diabetes Institute, Li Ka Shing Centre for Health Research Innovation, Edmonton, AB, Canada

3 Faculty of Kinesiology, Sport and Recreation, University of Alberta, Edmonton, AB, Canada

4 Women and Children's Health Research Institute, University of Alberta, Edmonton, AB, Canada

5 Department of Medicine, Cumming School of Medicine, Faculty of Medicine, University of Calgary, Calgary, AB, Canada

6 Department of Cardiac Sciences, Cumming School of Medicine, Faculty of Medicine, University of Calgary, Calgary, AB, Canada

7 Department of Community Health Sciences, Cumming School of Medicine, Faculty of Medicine, University of Calgary, Calgary, AB, Canada

8 Faculty of Kinesiology, University of Calgary, Calgary, AB, Canada authorities, or are extrapolated recommendations from stronger evidence.

There are, however, instances where the evidence suggests that individuals with type 1 diabetes should be classified differently and/or that different advice should be provided than suggested by the authors of the position statement. The authors state that inactive individuals may face an increased risk of exercise-induced hypoglycaemia, presumably assuming that frequent exercisers have more experience in managing insulin use and carbohydrate intake around exercise. While the study they cite to support this statement ([2]) did find this for severe hypoglycaemia involving coma (which has a frequency of $6.31 / 100$ person-years), for severe hypoglycaemia requiring assistance, which is more common (frequency: 23.52/100 person-years), risk of exercise-induced hypoglycaemia was significantly higher in more active women (all ages) and in all active participants aged 4580 years, as compared with inactive individuals [2]. A secondary analysis ( $n=44$ individuals with type 1 diabetes) also found that more aerobically fit (and, therefore, likely more active) individuals $(n=23)$ experienced greater declines in plasma glucose during aerobic exercise compared with those with poor fitness $(n=21)(-4.6 \pm 3.4 \mathrm{mmol} / \mathrm{l}$ vs $-2.1 \pm$ $3.1 \mathrm{mmol} / 1 ; p=0.02$ ) and, thus, had greater hypoglycaemia risk [3]. Therefore, the category of 'currently minimally exercising and/or high risk of hypoglycaemia', which was recommended by the authors of the position statement as part of a list of categories for individuals with type 1 diabetes [1], may merit rethinking.

Evidence indicating possible sex- and/or gender-related differences in risk of exercise-related hypoglycaemia may also deserve acknowledgement. Bohn et al. [2] found that the rate of severe hypoglycaemia requiring assistance was higher in physically active women with type 1 diabetes (all ages) than in those who were inactive, whereas the opposite relationship 
was found among men aged 18-45 years. Concerning lesssevere hypoglycaemia, a smaller observational study [4] found that male adolescents with type 1 diabetes had a higher risk of nocturnal hypoglycaemia [OR 3.14 (95\% CI 1.16, 8.47)] following afternoon moderate-to-vigorous physical activity than female participants. Similarly, Brockman et al's secondary analysis [5] found that adult male participants with type 1 diabetes experienced significant plasma glucose declines during resistance exercise (from $8.6 \pm 2.5 \mathrm{mmol} / 1$ pre-exercise to $6.3 \pm 2.1 \mathrm{mmol} / 1$ post-exercise; $p=0.001$ ) while female participants did not $(7.2 \pm 1.3 \mathrm{mmol} / \mathrm{l}$ preexercise to $7.3 \pm 1.3 \mathrm{mmol} / 1$ post-exercise; $p=0.99$ ). In addition, more male participants $(50 \%)$ than female participants $(33 \%)$ experienced nocturnal hypoglycaemia after exercise [5]. It is uncertain whether these data represent true sex-related differences in blood glucose responses to exercise, or whether these outcomes reflect higher levels of aerobic fitness (potentially associated with higher exercise-related hypoglycaemia risk [3]) among male individuals, or higher lean body mass causing greater skeletal-muscle glucose transport during and after exercise. Regardless, if blood glucose declines less in female individuals than male individuals with type 1 diabetes during exercise, the carbohydrate consumption recommendations may be excessive for females.

The statement that intense aerobic and anaerobic exercise and exercises with a load-profile similar to interval exercise stabilise or increase glucose levels' [1] is used to justify the recommendation of an insulin correction bolus before beginning resistance exercise or high-intensity interval exercise (HIIE). However, the literature selected to support this advice for HIIE [6-9] does not represent the full range of evidence available. One of the papers cited (where exercise was performed in the fed state [9]) measured a mean decline in capillary glucose levels of $-6.83 \mathrm{mmol} \mathrm{l}^{-1} \mathrm{~h}^{-1}$ during exercise; this simply does not support the statement being made. In the remaining studies cited, in which participants performed exercise in the fasting state, blood glucose increased during HIIE. There are also several studies not cited, in which participants were in a postprandial state [10-14], showing significant (up to $-5.0 \mathrm{mmol} / 1$ in one study [13]) decreases in blood glucose during this type of activity. The influence of prandial state on blood glucose responses to this type of activity has recently been highlighted in a small, repeated-measures study [15]. When 12 participants with type 1 diabetes performed morning (fasted) HIIE, an upward trend in capillary glucose (from $7.6 \pm 1.4 \mathrm{mmol} / \mathrm{l}$ to $9.2 \pm 2.9 \mathrm{mmol} / \mathrm{l}$ ) was observed, but the same HIIE protocol performed in the afternoon with the same participants in a postprandial state resulted in a decreasing trend in capillary glucose levels (from $9.9 \pm 3.1$ to $9.5 \pm 3.4 \mathrm{mmol} / \mathrm{l} ; p=0.014$, time $\times$ treatment interaction) [15].
The evidence also does not clearly support the suggestion that those performing resistance exercise should expect to see rising blood glucose levels. The one small study $(n=8)$ cited in the position statement [1] that supports this premise, by Turner et al. (2016) [16], involved participants with type 1 diabetes exercising while fasted. Consequently, the reported blood glucose increase $(+1.5 \pm 0.8 \mathrm{mmol} / \mathrm{l})$ over the course of a moderate resistance exercise protocol (three sets of eight repetitions) [16] is not surprising. The authors of the position statement [1], however, failed to cite studies of afternoon postprandial exercise by Farinha et al. $(n=9)$ [9], in which capillary blood glucose levels fell during resistance exercise sessions by approximately $-3 \mathrm{mmol} / \mathrm{l}$ (estimated from Fig. 1 of the publication by Farinha et al. [9]), and by our group ( $n=$ 12) [17], in which plasma glucose decreased from $8.4 \pm$ $2.7 \mathrm{mmol} / \mathrm{l}$ to $6.8 \pm 2.3 \mathrm{mmol} / \mathrm{l}(p=0.008)$ during resistance exercise. Notably, the studies by Turner et al. [16], Farinha et al. [9] and our group [17] used very similar resistance exercise protocols (with three sets of eight repetitions), both in terms of the exercises used and the intensity of the exercises [17]. Similar to HIIE, there is also a recent, small, crossover study [18] showing distinct patterns of response to a standardised resistance exercise protocol to compare morning (fasting) vs afternoon (postprandial) exercise: plasma glucose declined from $8.2 \pm 2.5 \mathrm{mmol} / 1$ to $7.4 \pm 2.6 \mathrm{mmol} / \mathrm{l}$ during afternoon, postprandial exercise, compared with an increase in plasma glucose from $9.5 \pm 3.0 \mathrm{mmol} / 1$ to $10.4 \pm 3.0 \mathrm{mmol} / \mathrm{l}$ $(p=0.031$, time $\times$ treatment interaction $)$ in the same participants during morning (fasted) exercise.

While some omissions may have been needed to meet journal requirements, the absence of this literature may have provided an incomplete picture of the impact of HIIE and resistance exercise on blood glucose in individuals with type 1 diabetes. Glycaemic responses may vary systematically by sex and by prandial state. We concede that blood glucose responses to exercise are variable in this population and that a correction dose of insulin before high-intensity activities may be appropriate for some (most likely young, fit, male individuals) [19] but probably not for all. We also appreciate that these recommendations are to be 'used as an initial guidance tool that also needs to be tailored individually' [1]. However, there is evidence to suggest that the recommendation to administer a correction dose of insulin prior to HIIE or resistance exercise may be most appropriate when exercising in a fasting state and, in the interest of the safety of individuals with type 1 diabetes, this clarification should be made.

Funding JEY is supported by a Heart and Stroke Foundation of Canada Alberta New Investigator Award.

Authors' relationships and activities JEY has received in-kind research contributions from Abbott Nutrition and LifeScan, along with speaker's fees and in-kind research contributions from Dexcom. RJS has received honoraria from Novo-Nordisk for attending advisory board 
meetings, and payments from Novo-Nordisk and Sanofi for being a local Principal Investigator in multicentre trials.

Contribution statement JEY and RJS both contributed to the writing, editing and final approval of the manuscript.

\section{References}

1. Moser O, Riddell MC, Eckstein ML et al (2020) Glucose management for exercise using continuous glucose monitoring (CGM) and intermittently scanned CGM (isCGM) systems in type 1 diabetes: Position statement of the European Association for the Study of diabetes (EASD) and of the International Society for Pediatric and Adolescent Diabetes (ISPAD) endorsed by JDRF and supported by the American Diabetes Association (ADA). Diabetologia 63:25012520. https://doi.org/10.1007/s00125-020-05263-9

2. Bohn B, Herbst A, Pfeifer M et al (2015) Impact of physical activity on glycemic control and prevalence of cardiovascular risk factors in adults with type 1 diabetes: A cross-sectional multicenter study of 18,028 patients. Diabetes Care 38(8):1536-1543. https://doi.org/ $10.2337 / \mathrm{dc} 15-0030$

3. Al Khalifah RA, Suppere C, Haidar A, Rabasa-Lhoret R, Ladouceur M, Legault L (2016) Association of aerobic fitness level with exercise-induced hypoglycaemia in type 1 diabetes. Diabet Med 33(12):1686-1690. https://doi.org/10.1111/dme.13070

4. Metcalf KM, Singhvi A, Tsalikian E et al (2014) Effects of moderate-to-vigorous intensity physical activity on overnight and next-day hypoglycemia in active adolescents with type 1 diabetes. Diabetes Care 37(5):1272-1278. https://doi.org/10.2337/dc131973

5. Brockman NK, Sigal RJ, Kenny GP, Riddell MC, Perkins BA, Yardley JE (2020) Sex-related differences in blood glucose responses to resistance exercise in adults with type 1 diabetes: A secondary data analysis. Can J Diabetes 44(3):267-273. https://doi. org/10.1016/j.jcjd.2019.08.006

6. Scott SN, Cocks M, Andrews RC et al (2019) Fasted high-intensity interval and moderate-intensity exercise do not Lead to detrimental 24-hour blood glucose profiles. J Clin Endocrinol Metab 104(1): 111-117. https://doi.org/10.1210/jc.2018-01308

7. Riddell MC, Pooni R, Yavelberg L et al (2019) Reproducibility in the cardiometabolic responses to high-intensity interval exercise in adults with type 1 diabetes. Diabetes Res Clin Pract 148:137-143. https://doi.org/10.1016/j.diabres.2019.01.003

8. Harmer AR, Chisholm DJ, McKenna MJ et al (2007) Highintensity training improves plasma glucose and acid-base regulation during intermittent maximal exercise in type 1 diabetes. Diabetes Care 30(5):1269-1271. https://doi.org/10.2337/dc061790
9. Farinha JB, Boff W, Dos Santos GC et al (2019) Acute glycemic responses along 10-week high-intensity training protocols in type 1 diabetes patients. Diabetes Res Clin Pract 153:111-113. https://doi. org/10.1016/j.diabres.2019.06.001

10. Guelfi KJ, Jones TW, Fournier PA (2005) The decline in blood glucose levels is less with intermittent high-intensity compared with moderate exercise in individuals with type 1 diabetes. Diabetes Care 28(6):1289-1294. https://doi.org/10.2337/diacare.28.6.1289

11. Maran A, Pavan P, Bonsembiante B et al (2010) Continuous glucose monitoring reveals delayed nocturnal hypoglycemia after intermittent high-intensity exercise in nontrained patients with type 1 diabetes. Diabetes Technol Ther 12(10):763-768. https://doi.org/ 10.1089/dia.2010.0038

12. Campbell MD, West DJ, Bain SC et al (2015) Simulated games activity vs continuous running exercise: A novel comparison of the glycemic and metabolic responses in T1DM patients. Scand J Med Sci Sports 25(2):216-222. https://doi.org/10.1111/sms. 12192

13. Iscoe KE, Riddell MC (2011) Continuous moderate-intensity exercise with or without intermittent high-intensity work: Effects on acute and late glycaemia in athletes with type 1 diabetes mellitus. Diabet Med 28(7):824-832. https://doi.org/10.1111/j.1464-5491. 2011.03274.x

14. Tonoli C, Heyman E, Roelands B et al (2015) BDNF, IGF-I, glucose and insulin during continuous and interval exercise in type 1 diabetes. Int J Sports Med 36(12):955-959. https://doi.org/10. 1055/s-0035-1548886

15. Yardley JE (2020) Fasting may alter blood glucose responses to high intensity interval exercise in adults with type 1 diabetes: A randomized acute crossover study. Can J Diabetes 44(8):727-733. https://doi.org/10.1016/j.jcjd.2020.09.007

16. Turner D, Gray BJ, Luzio S et al (2016) Similar magnitude of postexercise hyperglycemia despite manipulating resistance exercise intensity in type 1 diabetes individuals. Scand J Med Sci Sports 26(4):404-412. https://doi.org/10.1111/sms. 12472

17. Yardley JE, Kenny GP, Perkins BA et al (2013) Resistance versus aerobic exercise: Acute effects on glycemia in type 1 diabetes. Diabetes Care 36(3):537-542. https://doi.org/10.2337/dc12-0963

18. Toghi-Eshghi SR, Yardley JE (2019) Morning (fasting) vs afternoon resistance exercise in individuals with type 1 diabetes: A randomized crossover study. J Clin Endocrinol Metab 104(11): 5217-5224. https://doi.org/10.1210/jc.2018-02384

19. Yardley JE, Brockman NK, Bracken RM (2018) Could age, sex and physical fitness affect blood glucose responses to exercise in type 1 diabetes? Front Endocrinol 9:674. https://doi.org/10.3389/ fendo.2018.00674

Publisher's note Springer Nature remains neutral with regard to jurisdictional claims in published maps and institutional affiliations. 